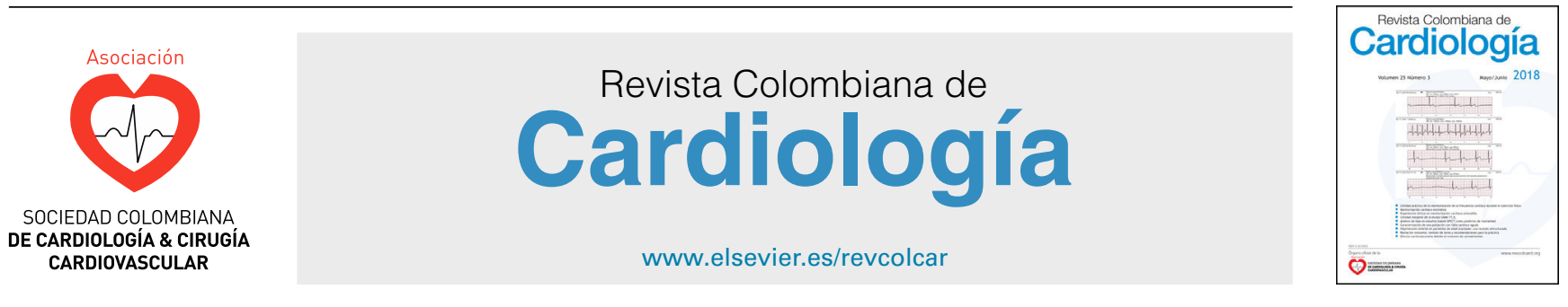

CARDIOLOGÍA DEL ADULTO - ARTÍCULO ORIGINAL

\title{
Estimación de los costos directos de los eventos coronarios en Colombia
}

\author{
Martín Romero*, Eliana Vásquez, Germán Acero y Lina Huérfano
}

\author{
Fundación Salutia - Centro de investigaciones en Salud, Bogotá, Colombia
}

Recibido el 6 de septiembre de 2017; aceptado el 4 de mayo de 2018

Disponible en Internet el 9 de octubre de 2018

\section{PALABRAS CLAVE \\ Infarto agudo de \\ miocardio; \\ Análisis de costos; \\ Diabetes mellitus; \\ Hipertensión; \\ Síndrome coronario \\ agudo.}

\begin{abstract}
Resumen
Objetivo: estimar los costos de atención en salud relacionados con los pacientes que desarrollan eventos coronarios asociados a factores de riesgo (hipertensión, diabetes e hipercolesterolemia), así como el costo de acuerdo con el tiempo de evolución luego de dicho evento, en el contexto colombiano.

Métodos: se llevó a cabo un estudio retrospectivo descriptivo a través de las bases de datos de suficiencia UPC de dos aseguradoras de salud colombianas, en las cuales se identificaron los pacientes que, durante el año 2014, presentaron algún evento coronario y se confirmaron sus antecedentes médicos (factores de riesgo) en la base del año 2013. Se estableció la tasa de presentación de eventos coronarios en la población y se extrajo el consumo reportado asociado a los procedimientos practicados durante un periodo de un año. Los costos se organizaron por categorías en diferentes cortes de tiempo desde la presentación del evento.

Resultados: se encontraron 2.103 pacientes con eventos coronarios de los cuales, como antecedente médico, 583 presentaban hipertensión arterial, 354 dislipidemia y 198 diabetes. El costo total promedio al año del evento coronario fue de COP\$23.454.569,95, sin factor de riesgo diagnosticado previamente fue de COP\$23.848.023,32 y con antecedentes médicos tratados fue de COP\$17.040.335,95, COP\$22.075.708,19 y COP\$28.825.710,71 para dislipidemia, hipertensión arterial y diabetes, respectivamente.

Conclusiones: un paciente sin diagnóstico temprano de enfermedades o factores de riesgo cardiovascular, al momento de presentar un evento coronario, tiene mayor costo en el manejo terapéutico del evento que aquel diagnosticado previamente y que recibe tratamiento para ello.

(c) 2018 Sociedad Colombiana de Cardiología y Cirugía Cardiovascular. Publicado por Elsevier España, S.L.U. Este es un artículo Open Access bajo la licencia CC BY-NC-ND (http:// creativecommons.org/licenses/by-nc-nd/4.0/).
\end{abstract}

\footnotetext{
* Autor para correspondencia.

Correo electrónico: martin.romero@salutia.org (M. Romero).
} 


\section{KEYWORDS}

Acute myocardial

infarction;

Cost analysis;

Diabetes mellitus;

Hypertension;

Acute coronary

syndrome.

\section{Estimation of the direct costs of coronary events in Colombia}

\begin{abstract}
Objective: To estimate the health care costs associated with patients that develop coronary events associated with risk factors (hypertension, diabetes, and hypercholesterolaemia), as well as the cost as regards the time of onset after such event, in the Colombian context.

Methods: A retrospective, descriptive study was conducted using the Per-capita Payment Unit (UPC) sufficiency databases of two Colombian insurance companies. Patients were identified that presented with a cardiac event during the year 2014, and their medical history was confirmed (risk factors). The rate of presenting with coronary events in the population was established and the reported costs associated with the procedures practiced during a one-year period were extracted. The costs were organised by categories into different time cut-offs from the presentation of the event.

Results: A total of 2,103 patients were found with coronary events, of which 583 had arterial hypertension, 354 with dyslipidaemia, and 198 patients with diabetes, as medical history. The total average cost a year of the coronary event was (COP\$23,454,569.95), with no previously diagnosed risk it was (COP $\$ 23,848,023.32$ ), and with a treated medical history it was (COP\$17,040,335.95), (COP\$22,075,708.19) and (COP\$28,825,710.71) for dyslipidaemia, arterial hypertension, and diabetes, respectively.

Conclusions: A patient without an early diagnosis of cardiovascular diseases or risk factors, on presenting with a coronary event, incurs higher costs in the therapeutic management of the event than one previously diagnosed and receives treatment for it.

(c) 2018 Sociedad Colombiana de Cardiología y Cirugía Cardiovascular. Published by Elsevier España, S.L.U. This is an open access article under the CC BY-NC-ND license (http:// creativecommons.org/licenses/by-nc-nd/4.0/).
\end{abstract}

\section{Introducción}

Se estima que las enfermedades cardiovasculares son las causantes del $28 \%$ de las muertes en países de medianos y bajos ingresos ${ }^{1}$. En Latinoamérica, en 2001 esta enfermedad fue la causante del $31 \%$ de todas las muertes ${ }^{1}$ y se prevé que el número de casos aumente debido a la transición epidemiológica que vive la región, además de la alta carga de factores de riesgo que tiene la población.

El estudio INTERHEART, que involucra 6 países latinoamericanos incluido Colombia, mostró que factores de riesgo como la hipertensión tienen una asociación fuerte con el evento coronario agudo, presentándose en el $32,9 \%$ de la población con riesgo atribuible ${ }^{2}$.

En el informe sobre la situación actual en salud 2014 de Colombia $^{3}$, la enfermedad isquémica del corazón se posicionó como una de las principales causas de muerte en las personas mayores de 45 años o más ${ }^{4}$, seguida de las enfermedades cerebrovasculares, representando tasas de muerte de 75,58 y 32,74 por 100.000 habitantes, respectivamente ${ }^{3}$; en el desarrollo de la enfermedad están implicados numerosos factores de riesgo, como los trastornos del metabolismo de los lípidos (dislipidemias) ${ }^{5}$, la hipertensión y la diabetes. Estudios como el de Yusuf et al. ${ }^{6}$, desarrollado en 52 países, identificó los factores de riesgo modificables en los pacientes que presentaron infarto agudo de miocardio, entre los cuales se encontraba: tabaquismo, dislipidemia, hipertensión arterial, diabetes mellitus, obesidad mórbida, sedentarismo, entre otros.

Aunque se sabe que existe una variedad de factores de riesgo para que una persona presente un evento coronario, dentro de los controlables o prevenibles están los trastornos del metabolismo de los lípidos (dislipidemias), hipertensión y diabetes que, además, son considerados como los de mayor riesgo $^{6}$, afirmación avalada también por el estudio de Rodríguez et al. ${ }^{7}$, que tuvo como objetivo identificar la presencia de factores de riesgo modificables en pacientes que presentaron síndrome coronario agudo, determinando que los cuatro factores principales fueron HTA, tabaquismo, dislipidemia y diabetes.

La enfermedad cardiaca aguda genera un alto impacto económico en los sistemas de salud, debido al uso de recursos para la atención de los eventos y sus complicaciones, además porque el paciente que sufre un evento se encuentra frente al riesgo de presentar un nuevo evento que aumenta los costos de manejo de la enfermedad y el control de las eventuales complicaciones consumiendo una cantidad importante de recursos por largo plazo. Un estudio desarrollado en España encontró que el costo medio por paciente con diagnóstico de síndrome coronario agudo fue de 14.069 euros; este costo incluía la pérdida de productividad ${ }^{8}$, las atenciones especializadas, la hospitalización y los costos de atención primaria, pero no mencionaba los costos tardíos asociados a la enfermedad.

Partiendo de la necesidad de contar con datos reales para el país, relacionados con el costo de atención del paciente con evento coronario agudo, se desarrolló un análisis de costos en pacientes que han sufrido este evento con o sin diagnóstico previo de factores de riesgo como dislipidemia, hipertensión arterial o diabetes, a fin de evaluar esos costos totales y por categorías con el objetivo de determinar si existe diferencia respecto a cada subgrupo, especialmente 
en aquellos que en el momento de presentar el evento coronario no tuvieron tratamiento previo para alguno de los factores de riesgo cardiovascular.

\section{Método}

Se realizó un estudio retrospectivo descriptivo, que partió de la revisión de las bases de datos de consumos en servicios de salud reportados en aseguradoras colombianas, con el fin de identificar los pacientes que presentaron eventos coronarios y los costos de atención asociados.

Para el desarrollo de este estudio se discriminaron antecedentes de dislipidemia, hipertensión arterial y diabetes, teniendo en cuenta que según la literatura antes descrita, son tres de los factores de riesgo más frecuentes para desarrollar un evento coronario y que por las características de los registros, sería posible identificarlos.

De acuerdo con lo anterior, se seleccionaron usuarios con evento coronario en el primer semestre del año 2014, los cuales fueron seguidos en sus costos por un periodo mínimo de un año o hasta su muerte. Sobre esta población, se identificaron pacientes con HTA, dislipidemia o diabetes según registros del segundo semestre de 2013 (hasta seis meses antes del evento). Los pacientes que no tenían un diagnóstico previo de alguno de los factores de riesgo fueron catalogados dentro del análisis como pacientes con o sin diagnóstico de factores de riesgo, los cuales fueron comparados con el costo del paciente que presentaba un diagnóstico previo de alguno de los factores de riesgo analizados.

\section{Selección de los pacientes}

Por la complejidad de la información y la calidad de los datos, la identificación de los pacientes con evento coronario, fue definida mediante el diagnóstico de egreso hospitalario según código de CIE-10 (I200, I210, I211, I212, 1213, 1214, 1219, 1220, 1221, 1228, 1229, 1248, 1249, 1460, 1461 y 1469) que corresponde a los códigos asociados a evento coronario agudo y validados con la presencia en los registros de códigos de procedimientos, CUPS, asociados a atenciones inherentes a estos eventos de manera intrahospitalaria (360100, 360101, 360102, 360200, 360201, 360202, $360300,360400,360401,360500,360600,361000,361100$, $361200,361300$ y 361400$)$. Este procedimiento permitió la eliminación de pacientes que no tenían relacionado ningún procedimiento con el diagnóstico de interés identificado.

Una vez identificados los pacientes con diagnósticos de interés y verificados sus consumos, se procedió a revisar la base da datos del año anterior (2013), con el fin de identificar a los pacientes que, previo al evento, tenían un diagnóstico de factor de riesgo. La identificación de los factores de riesgo se realizó mediante los códigos CIE-10 E780, E781, E782, E784, E785, E788 y E789 para dislipidemia, para HTA únicamente I10 y para diabetes los códigos CIE-10 E10, E11, E12, E13 y E14. Para la caracterización de estos riesgos, se utilizó el algoritmo mencionado por Romero et al. 9,10 (presentar tres o más registros de consulta y un registro de egreso de la enfermedad), en el periodo de análisis, con el fin de que aquellos pacientes que cumplieran fueran identificados con ese antecedente. De igual manera, y teniendo en cuenta la gran cantidad de subregistro, se confirmó que tuvieran dispensación de medicamentos asociados a cada uno de los factores de riesgo de interés por al menos tres meses consecutivos.

\section{Información técnica}

\section{Costos}

Para el cálculo de los costos del manejo médico del evento coronario, se buscó en las bases de datos analizadas los servicios utilizados por estos pacientes, incluyendo servicios, insumos, medicamentos y dispositivos, relacionados por categorías: consultas a especialistas, consultas domiciliarias, urgencias, procedimientos quirúrgicos y no quirúrgicos, medicamentos, exámenes de laboratorio, terapias, imágenes diagnósticas, servicio de salas de cirugía, traslados, hospitalización e insumos (incluido entre ellos el stent). Dichos códigos fueron validados por dos investigadores profesionales de la salud, con el fin de validar que sí estuvieran relacionados con el evento de interés. Se excluyeron atenciones prestadas por servicios no asociados a la patología como eventos traumáticos, eventos infecciosos, cáncer y otros.

Para el análisis de los costos directos de eventos cardiovasculares, se establecieron 4 cortes de tiempo, desde el día de presentación del evento hasta el día 30, luego del día 31 hasta el día 90; desde el día 91 hasta el 180 y finalmente se analizó el costo promedio desde el día 181 hasta el día 360.

Los costos fueron analizados de manera global y por categorías frente a las diferencias en pacientes con factores de riesgo identificados previamente o no. Los datos extremos fueron revisados por dos expertos clínicos, quienes tenían la facultad de determinar la permanencia o exclusión por consistencia o inconsistencia en los consumos registrados.

\section{Resultados}

\section{Pacientes}

Se identificaron 2.103 pacientes, de una población analizada de 7.965.170 afiliados al sistema de salud, lo que da una tasa de presentación de evento cardiovascular de 0,26 por 1.000 personas. Se encontró que el 72,8\% eran hombres con edad promedio de 64 años (tabla 1); no obstante, 69 pacientes

Tabla 1 Características de la población analizada

\begin{tabular}{ll}
\hline Variable & Valor \\
\hline Edad promedio & 65 años (IC 95\%: 44 - 96) \\
Hombres & $1.532(72,8 \%)$ \\
Mujeres & $571(28,2 \%)$ \\
Región de procedencia & \\
$\quad$ Amazónica & $2(0,35 \%)$ \\
Andina & $435(76,05 \%)$ \\
Caribe & $69(12,64 \%)$ \\
Llanos orientales & $24(4,20 \%)$ \\
Pacífica & $42(7,34 \%)$ \\
\hline
\end{tabular}

Fuente: elaboración de los autores, 2016. 
Tabla 2 Antecedentes médicos (factores de riesgo)

\begin{tabular}{lll}
\hline Co-morbilidad & $\mathrm{n}$ & $\%$ del total \\
\hline Dislipidemia & 20 & $3,50 \%$ \\
HTA & 126 & $22,03 \%$ \\
Diabetes & 21 & $3,67 \%$ \\
2 enfermedades (dislipidemia e HTA) & 105 & $18,36 \%$ \\
2 enfermedades (HTA y diabetes) & 21 & $3,67 \%$ \\
2 enfermedades (dislipidemia y diabetes) & 4 & $0,70 \%$ \\
3 enfermedades & 54 & $0,94 \%$ \\
Sin factor de riesgo diagnosticado & 220 & $38,46 \%$ \\
\hline
\end{tabular}

Fuente: estimación a partir de base de datos de consumos del año 2013 en pacientes seleccionados, 2016.

Tabla 3 Costos directos del evento coronario

\begin{tabular}{lllll}
\hline Indicador & Promedio COP & Mediana COP & Min COP & Max COP \\
\hline Mujeres & $\$ 17.217 .561,20$ & $\$ 15.781 .432$ & $\$ 6.897 .908$ & $\$ 54.101 .920$ \\
Hombres & $\$ 19.206 .748,16$ & $\$ 16.842 .021$ & $\$ 6.691 .270$ & $\$ 56.867 .519$ \\
TOTAL & $\$ 18.442 .956,51$ & $\$ 18.408 .841,39$ & $\$ 6.691 .270$ & $\$ 54.983 .882$ \\
\hline
\end{tabular}

Fuente: estimación a partir de la base de datos de consumos 2014.

con registros de evento coronario tenían menos de 40 años, los cuales representan el 3,28\% de la población de estudio.

Además, el $52,87 \%$ de los pacientes tenía consultas previas por alguno de los tres factores de riesgo estudiados en este análisis (diabetes, hipertensión, dislipidemias), de los cuales el $22,73 \%$ presentaba dos de los factores, el $0,94 \%$ tres factores y el $38,46 \%$ correspondió a pacientes sin factor de riesgo diagnosticado previamente (tabla 2 ).

\section{Costos}

\section{Costos del evento coronario agudo (día cero al día 30)}

El costo promedio total de un paciente, en el momento del evento coronario, en el primer mes fue de COP\$18.442.956,51, siendo mayor en hombres (COP\$19.206.748,16), con un IC (COP\$17.706.284,99 COP\$19.179.627,01) sin diferencias significativas (tabla 3).

Al evaluar este costo por categorías, se identificó que los insumos, incluidos los dispositivos médicos y los procedimientos quirúrgicos, representaron aproximadamente el $62,86 \%$ del costo total, seguido por la estancia hospitalaria en el $15,81 \%$; el discriminado total se puede ver en la figura 1.

\section{Costos posteriores al evento coronario}

Este fue disminuyendo, estabilizándose en cerca de COP\$200.000 mensuales a partir del segundo semestre tal y como se muestra en la tabla 4.

Para los siguientes 60 días al evento (del día 31 hasta el día 90), se registró un costo promedio de COP\$2.041.859,10 (IC 95\%: COP \$1.571.002,27 - COP \$2.512.715,93), equivalente a COP\$1.020.929,55 por mes en promedio. Durante el periodo del día 91 al día 180 (90 días), el costo promedio fue de COP $\$ 1.771 .011,11$, (IC 95\%: COP $\$ 1.325 .827,85$ - COP $\$ 2.216 .194,38)$, correspondiente a COP\$590.337,04 por mes. Finalmente, durante el periodo a partir del día 181 hasta el 360, se registró un costo promedio de manejo de COP\$1.198.743,74 (IC 95\%: COP\$880.855,32 COP\$1.516.632,16), correspondiente a COP\$199.790,62 por mes.

De acuerdo con lo anterior, el costo total promedio en un año de un paciente que presentó un evento coronario fue de COP\$23.454.569,95 con un IC del 95\% (\$ 22.961.929,76 COP - \$23.947.210,15 COP); fue mayor el costo incurrido en los primeros treinta días $(78,63 \%$ del costo total), seguido del $8,71 \%$ en los dos meses posteriores al evento, del $7,55 \%$ de los 3 a 6 meses y del 5,11\% de los 6 a 12 meses posteriores al evento (fig. 2).

Al comparar los diferentes resultados por sexo, en todos los casos, las mujeres presentaron un mayor costo que los hombres; el costo total general fue de COP\$24.710.029,82 (IC 95\%: COP\$18.874.455,31 - \$ 30.545.604,33) frente a $\$ 23.690 .452,24$ COP (IC 95\%: 21.195.474,9 - COP\$26.185.429,57) aunque no representó diferencias significativas.

\section{Costos de pacientes con antecedentes de enfermedades}

Al diferenciar los costos en pacientes con y sin riesgo identificado, se hallaron diferencias por tipo de pacientes; los de mayor costo fueron aquellos con antecedentes de diabetes y los de menor costo los que venían siendo tratados por dislipidemia (tabla 5).

Igualmente, se evidenció que los pacientes con múltiples riesgos serían más costosos, en especial cuando se combinan con diabetes. En los que presentaron las tres comorbilidades el costo promedio de atención del evento coronario por paciente fue de COP\$26.808.399 con un IC 95\% (COP\$22.430.048,70 - COP\$31.186.749,30), lo que indica que el costo va incrementándose al aumentar el riesgo. 


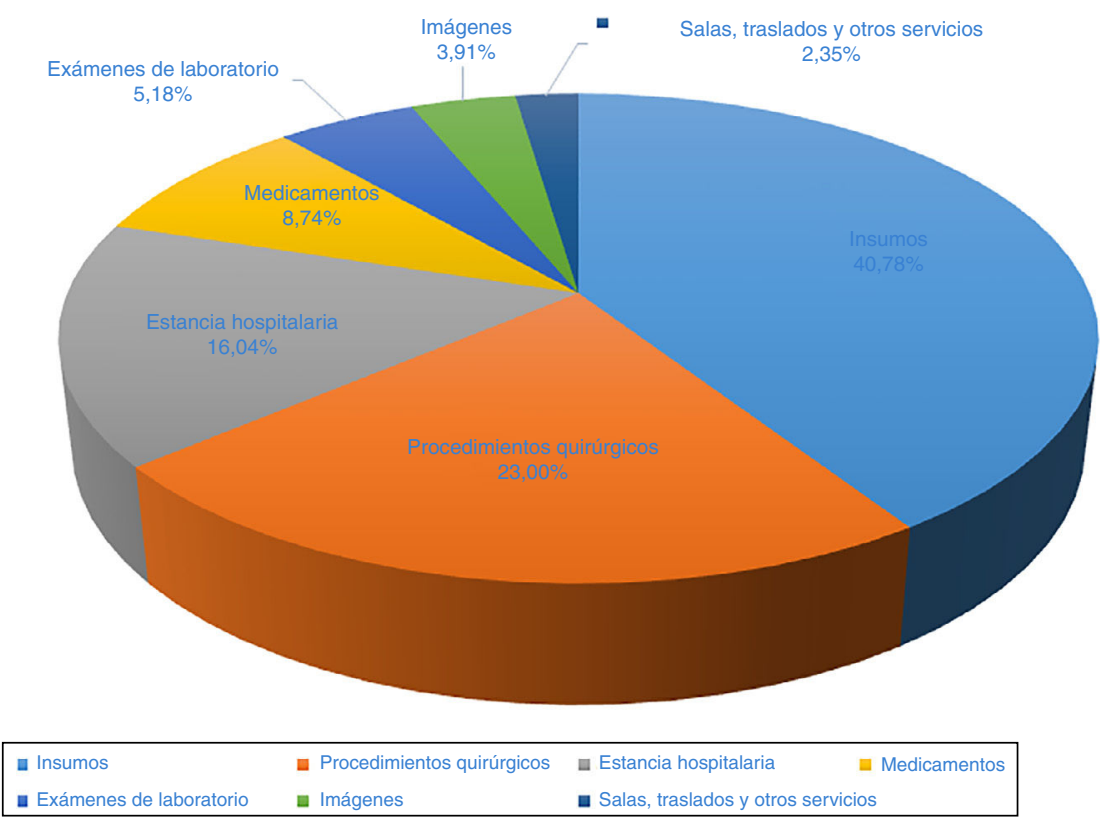

Figura 1 Distribución del costo (día cero al día 30) en el manejo del evento coronario.

Tabla 4 Costos de manejo posteriores al evento coronario (promedio por paciente en el periodo y mensual)

\begin{tabular}{llll}
\hline Indicador & $30-90$ días & $91-180$ días & $181-360$ días \\
\hline Femenino & $\$ 1.622 .086,28$ & $\$ 1.701 .767,24$ & $\$ 1.412 .906,7$ \\
Masculino & $\$ 2.133 .626,04$ & $\$ 1.961 .200,9$ & $\$ 1.120 .866,2$ \\
TOTAL & $\$ 2.041 .859,10$ & $\$ 1.771 .011,11$ & $\$ 1.198 .743,74$ \\
PROMEDIO MENSUAL & $\$ 1.020 .929,55$ & $\$ 590.337,04$ & $\$ 199.790,62$ \\
\hline
\end{tabular}

Fuente: estimado a partir de bases de datos de consumos para 2014 y 2015.

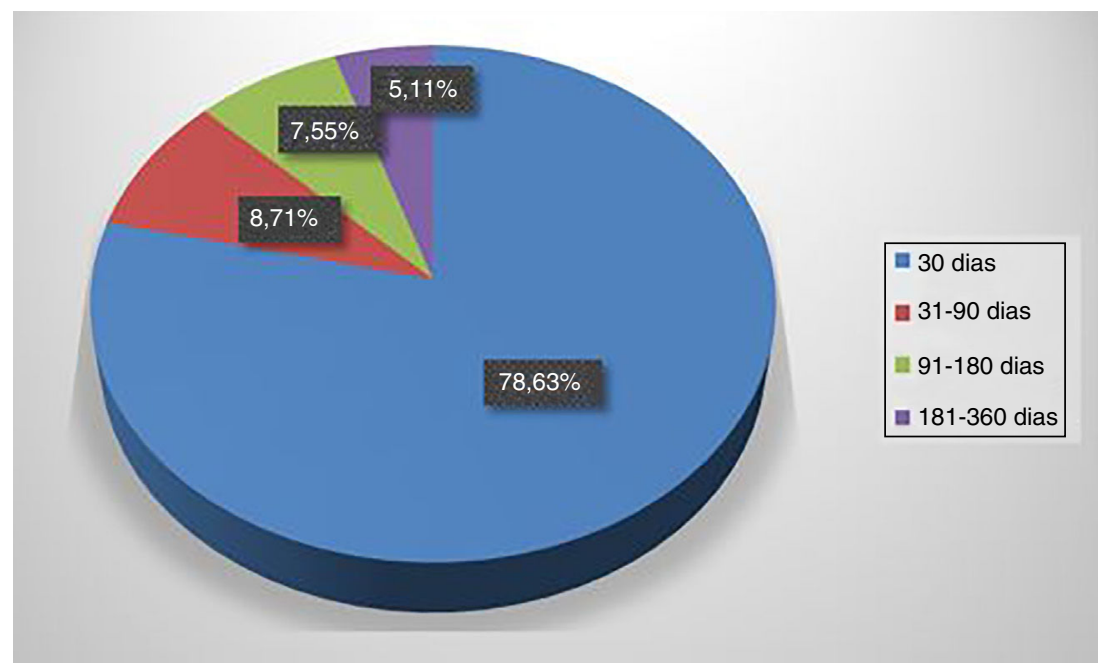

Figura 2 Distribución del costo total anual promedio por paciente con evento coronario.

\section{Discusión}

Este análisis muestra información muy valiosa para el sistema de salud colombiano, debido a que se analizó el $42,07 \%$ de la población total afiliada al régimen contributivo del país. Así mismo y debido a que existe ausencia de información relacionada con el costo de las enfermedades que más afectan a la población colombiana, este análisis establece, mediante datos reales, el costo promedio de un evento coronario en un año de seguimiento en diferentes 
Tabla 5 Costo promedio de los pacientes con evento coronario y antecedentes médico de riesgo

\begin{tabular}{lllll}
\hline Indicador & $\begin{array}{l}\text { Dislipidemia }(\mathrm{n} \\
\mathrm{n} 20)\end{array}$ & $\begin{array}{l}\text { Hipertensión } \\
\text { arterial }(\mathrm{n}=126)\end{array}$ & Diabetes $(\mathrm{n}=21)$ & $\begin{array}{l}\text { Sin factor de riesgo } \\
\text { diagnosticado }(\mathrm{n}=220)\end{array}$ \\
\hline Costo promedio anual & $\$ 17.040 .335,95$ & $\$ 22.075 .708,19$ & $\$ 28.825 .710,71$ & $\$ 23.848 .023,32$ \\
& $($ IC $95 \%)$ & $($ IC $95 \%)$ & $(I C 95 \%)$ & $($ IC $95 \%$ \\
& $14.313 .065-$ & $19.848 .189-$ & $20.556 .098-$ & $21.553 .926,53-$ \\
& $19.767 .606)$ & 24.303 .226 & 37.095 .322 & $26.142 .120,11)$ \\
\hline
\end{tabular}

Fuente: elaboración de los autores, 2016.

periodos de tiempo. Además, permite evidenciar el costo de los pacientes con factores de riesgo en tratamientos como dislipidemia, hipertensión arterial o diabetes y de aquellos que no presentan un antecedente de factor de riesgo diagnosticado previo al evento.

La principal limitación de este estudio radica en que la información proviene de bases de datos de aseguradoras y por tanto no es posible analizar más a fondo las condiciones y antecedentes médicos comprobables mediante historia clínica. Este análisis parte de los servicios usados por los pacientes y los diagnósticos asociados a las atenciones, siendo entonces un análisis de costos de la morbilidad atendida.

Se encontró que en Colombia, el costo directo promedio de un evento coronario durante los primeros treinta días es de COP\$18.750.682,45 (IC 95\% COP\$ 17.706.284COP\$19.179.627); este costo es el más alto de los cuatro periodos estudiados, dato que concuerda con el análisis previo realizado por Romero et al. ${ }^{11}$, en el que se evidenció un mayor costo durante el primer mes luego de presentado el evento.

Así mismo, el estudio de Henry et al. ${ }^{12}$, muestra cómo el costo del evento cardiovascular es mayor durante los primeros 30 días de seguimiento del mismo (periodo agudo), dato consecuente con los datos encontrados en este análisis. Por consiguiente, el costo del tratamiento del evento coronario después del periodo agudo, disminuye en más del $80 \%$, tal y como se menciona en los estudios de Henk et al. ${ }^{12}$, Brüggenjürgen et al. ${ }^{13}$ y Reinhold et al. ${ }^{14}$, los cuales refieren que el costo de los recursos usados en la fase aguda del evento son mayores a los usados con posterioridad.

Por otra parte, el costo del tratamiento del evento coronario en los pacientes con antecedentes de enfermedades como dislipidemia, HTA y diabetes fue en promedio de COP $\$ 17.040 .335,95$, COP\$22.075.708,19 y COP\$28.825.710,71 respectivamente, siendo significativa esta diferencia. Sin embargo, es importante mencionar que el costo encontrado en los pacientes con antecedente de dislipidemia y HTA puede estar disminuido en comparación con el costo promedio general de COP\$23.454.569,95, debido a que estos factores de riesgo se encuentran controlados con el tratamiento médico.

Además de lo anterior, los pacientes que no presentan un factor de riesgo previo diagnosticado al evento coronario registran un costo de COP\$23.848.023,32 (IC del 95\% COP\$21.553.926,53 -COP\$26.142.120,11), mayor en comparación con el costo promedio general de COP\$23.454.569,95 (IC del 95\% COP\$22.961.929,76 - COP\$23.947.210,15), lo que permite pensar que, si un paciente tiene factores de riesgo para evento coronario y no son diagnosticados previamente y tratados, al presentar el evento genera mayores costos que un paciente con factores de riesgo identificados y tratados previamente, como es el caso de aquellos con dislipidemias tratadas. Este estudio se puede considerar como el primer reporte de costos del evento coronario dentro del contexto colombiano, ya que no existen antecedentes de este tipo de análisis en el país.

\section{Conclusiones}

Se puede concluir que si una persona presenta enfermedades o factores de riesgo cardiovascular y no son diagnosticados y tratados previos a un evento coronario, este paciente registrará un mayor costo en el manejo terapéutico del evento comparado con aquellos que sí tuvieron un diagnóstico temprano y reciben tratamiento, demostrándose así la necesidad de contar con un tratamiento oportuno de los factores de riesgo asociados a la enfermedad cardiovascular.

\section{Bibliografía}

1. Hernández-Leiva E. Epidemiología del síndrome coronario agudo y la insuficiencia cardiaca en Latinoamérica. Rev Esp Cardiol. 2011;64:34-43.

2. Lanas F, Avezum A, Bautista LE, Diaz R, Luna M, Islam S, et al. Risk factors for acute myocardial infarction in Latin America: the INTERHEART Latin American study. Circulation. 2007;115:1067-74

3. Ministerio de Salud y Protección Social. Análisis de Situación de Salud - Colombia 2014 [Internet]. 2014 [Acceso 30 Aug 2017]. Disponible en: https://www.minsalud.gov.co/sites/ rid/Lists/BibliotecaDigital/RIDE/VS/ED/PSP/ASIS_2014_v11.pdf.

4. Sociedad colombiana de cardiología y cirugía cardiovascular. Guías colombianas de cardiología síndrome coronario agudo sin elevación del st (angina inestable e infarto agudo del miocardio sin elevación del ST) [Internet]. Re Colomb Cardiol.; 2008 [Acceso 7 Jul 2017]. Disponible en: http://scc.org.co/wpcontent/uploads/2012/08/8-guia-enf-coronaria-2008.pdf.

5. Machado-Alba JE, Machado-Duque ME. Cardiovascular risk factors prevalence among patients with dyslipidemia in Colombia. Rev Peru Med Exp Salud Pública. 2013;30:205-11.

6. Yusuf S, Hawken S, Ounpuu S, Dans T, Avezum A, Lanas F, et al. Effect of potentially modifiable risk factors associated with myocardial infarction in 52 countries (the INTERHEART study): case-control study. Lancet Lond Engl. 2004;364:937-52.

7. Rodríguez Navarro AY, et al. Factores de riesgo en pacientes con síndrome coronario agudo remitidos para coronariografía. CorSalud. 2013;5:280-4.

8. Fernández-de-Bobadilla J, López-de-Sá E. Carga económica y social de la enfermedad coronaria. Rev Esp Cardiol. 2013;13 Supl. B:42-7. 
9. Romero M, Marrugo Figueroa R, Benavides M, Alzate P, Reinales J, Quinche G, et al. Modelo de caracterización de una población afiliada a una asegurada en colombia, mediante una metodología de agrupación por riesgo. Value Health. 2015;18: A812.

10. Romero ME, Huerfano LM, Sanchez VO, Marrugo RD, Arango $\mathrm{CH}$, Sanabria $\mathrm{M}$, et al. Gestión territorial del riesgo en salud: herramienta metodológica para la toma de decisiones, Guía de modelo de monitoreo. Colombia: Fundación Salutia;; 2014.

11. Romero M, Torres A, Grosso G, Urrutia C. Cost analysis of patients with acute Coronary event in a Commercial Insurance Company in Colombia -Saludcoop- in 2013. Value Health. 2015;18:A256.
12. Henk HJ, Paoli CJ, Gandra SR. A Retrospective Study to Examine Healthcare Costs Related to Cardiovascular Events in Individuals with Hyperlipidemia. Adv Ther. 2015;32: 1104-16.

13. Brüggenjürgen $B$, Rupprecht $H-J$, Willich $S N$, Spannagl $M$, Ehlken B, Smala A, et al. Cost of atherothrombotic diseases-myocardial infarction, ischaemic stroke and peripheral arterial occlusive disease-in Germany. J Public Health. 20051;13(4):216-24.

14. Reinhold T, Lindig C, Willich SN, Brüggenjürgen B. The costs of myocardial infarction-a longitudinal analysis using data from a large German health insurance company. J Public Health. 2011;19:579-86. 